is an accessible one, such as cancer of the skin, cervix uteri, mouth, or breast. In addition, ingenious operations have been devised by which laryngeal and rectal growths can be made accessible. In most other regions it is not possible to subject a tumour to an adequate homogeneous $\gamma$ irradiation, unless perhaps in those few clinics which possess very large quantities of radium.

Radium may be placed against the surface of a growth, or needles containing it may be implanted into and around the tumour. Seeds are also used in this manner. In addition, in a few of the larger clinics teletherapy is available. In this method a large quantity of radium in the form of a bomb is held several centimetres from the body and used as a very powerful and inexhaustible $x$-ray tube. There is a fourth method of application-namely, cavitation, in which tubes containing radium are placed in the natural cavities of the body; but this is now little used, except in the treatment of cancer of the cervix uteri, when it is usuatly combined with surface therapy.

Implantation is the method which most concerns the surgeon, and the rest of radiotherapy (estimated by Forssell as about $\mathbf{7 5}$ per cent. of its entire scope) is of minor interest to him. It is peculiarly fitting that this method should be discussed in Dublin, for it was here that implantation originated. I refer to the work of Professor Joly and the late Dr. Stevenson, who were the first to introduce a tube of emanation into a metal needle. This marked one of the greatest advances in the treatment of cancer, and from it all modern implantation techniques have arisen. At present interstitial irradiation is a rather unscientific procedure, which is just as well carried out by a surgeon as by anyone else. Indeed, it can be argued that owing to his training a surgeon will insert needles with greater skill than a mere radiologist. It is likely, however, that as our knowledge of radiophysics and radiobiology increases, implantation will become just as exact a method of treatment as teletherapy or $x$-ray therapy, in which case only persons with special knowiedge will be capable of using it properly. That these will be radiologists seems obvious. Radium is only implanted in a few sites, and it seems likeiy that a radiotherapist, working in a special clinic and seeing many more malignant cases than the average surgeon, will develop an excellent technique even if he does not possess special surgical knowledge. $\mathrm{He}$ will also know when implantation should not be used and when other methods of application or deep $x$-ray therapy are preferable. Hence we may conclude that radium therapy will be best developed if it is left in the hands of trained specialists who devote their whole time to it, and who have the necessary lnowledge and training to keep abreast of the rapid advances which are taking place.

Poor results are frequently reported from the use of a technique which has been highly successful in other hands. This is because the result of treatment does not depend so much on the technique itself as on the intelligence with which it is employed. Not only must the treatment of each case be properly planned, but it must be carefully carried through with due regard to the reaction of the individual patient. In practice this is only possible in a properfy organized and completely equipped clinic directed by an experienced radiotherapist.

The demand for a special radiotherapeutic clinic may seem extravagant, but if one pictures a surgeon who has to operate on patients sent from other departments without having the opportunity of first making a diagnosis or of following up the result of his surgical treatment, one has some idea of the difficulties of the radiologist who does not work in a special department. Radiotherapeutic technique is no easier than that of surgery, and the results obtained by it and the harm it may do are only apparent after a considerable time. Therefore, like any other specialist, the radiologist must foliow up and study his cases. Each patient must be examined at regular and frequent intervals, not only for the good of the individual, but also so that the value of the treatment may be assessed. To do this, statistics must be carefully prepared and should be in such a form that they are not misleading and can be compared easily with those from other clinics. The following up of patients depends "on careful internal organization of the clinic, and close cooperation between it, the patient himself, his doctor, the staff of other hospitals, and the state medical authorities.

The foregoing remarks have been made much more interestingly many times before. Indeed, they may be described in Mr. Punch's words as " blinding glimpses of the obvious." But until they are recognized and acted upon, the obvious cannot too often be repeated.

\section{THE RADIATION TREATMENT OF MALIGNANT DISEASE*}

\author{
BY
}

WALTER M. LEVITT, M.B., M.R.C.P., D.M.R.E.

MEDICAL OFFICER IN CHARGE OF THE RADIOTHERAPEUTIC DFPAKTMENT, ST. BARTHOLOMEW'S HOSPITAL

The object of this discussion is, I suppose, to attempt to answer the question: When, and in what form, may radiations best be applied in the treatment of malignant disease? I propose, first of all, briefly to summarize the various methods of application of radiations, and then to consider the indications for their use.

\section{Methods of Application of Radiations}

Radiations are commonly used in the treatment of malignant disease as $x$ rays or as the gamma rays of radium and radon: (a) by interstitial methods, the radium or radon being inserted into the tissues enclosed in needles or "s seeds"; (b) by short-distance surface application, the radium being applied to the surface of the body on applicators or other apparatus moulded to the part; (c) intracavitary application, the radium being introduced in suitable containers into the natural body cavities; (d) by long-distance surface application, the so-called telecurie- or bomb-therapy, a large quantity of radium being massed in a single container, which is applied at a distance of several centimetres from the surface.

I would like to make it clear that wherever $x$ rays are mentioned in this paper I refer to high-voltage, heavily filtered rays as used in modern deep $x$-ray therapy. While it is true that there are still one or two radiologists who cling to the use of soft, non-penetrating radiations, even in the treatment of malignant disease, agreement is practically universal that it is only by the use of the hardest radiations that the best results are to be obtained.

The broad principles of the use of all these methods are well known and do not need recapitulation; what concerns us here is the relative value of the methods in the treatment of cancer. If we compare these methods of application of radiations it will soon become apparent that their scope is very different. If we may make the reasonable assumption that it is essential in the irradiation of every malignant growth to apply an adequate dose of radiation throughout the whole extent of the growth, we find, for example, that the proportion of malignant tumours that can be adequately treated by interstitial methods is very limited, principally by anatomical considerations limiting the surgical access to the growth for the purpose of inserting the needles. Again, in the case of short-distance radiotherapy the depth at which the requisite dosage can be obtained is limited to a few centi-

* Read in opening a discussion at a combined meeting of the Sections of Radiology and Surgery at the Annual Meeting of the British Medical Association, Dublin, 1933. 
metres, and, accordingly, only comparatively superficial growths lend themselves to this form of treatment. Similarly, the limitations of the intracavitary method are at once apparent, and in practice this method is used principally for the treatment of carcinoma of the uterus, and occasionally for growths in the neighbourhood of the tonsils and oesophagus. Both the radium bomb (assuming that sufficient radium is available) and $x$ rays of sufficient penetrating power theoretically permit of application by cross-fire methods of almost any desired dose to any region of the body, but in practice we find that where the disease is widespread the application of large doses is not possible.

We have now briefly examined the various methods of application of radiations from the point of view of technical efficiency or dosage. Before passing on to a consideration of their relative effectiveness in the treatment of malignant disease let us summarize the information we have obtained so far.

1. Interstitial radium.-Limited application to growths fairly localized and surgically accessible, but very intense local action obtainable.

2. Short-distance surface application of radium.-Intense local action for a limited depth only; can only be used therefore, for comparatively superficial growths except when combined with other methods.

3. Intracavitary application of radium.-Use practically limited to vagina, mouth, and throat.

4. $X$ rays and radium bomb.-Used in similar ways intense action can be produced in any desired region, but heavy dosage not tolerated in very extensive diséase.

If we wish to decide what should be the ideal form of radiological treatment for any growth (I am not now considering surgical treatment), it is obvious that at least equally important with the various methods of application available is the possession of certain special knowledge about the growth itself. The facts which we require to know are: (1) the origin and type of growth, as providing a guide to the probable radio-sensitivity; (2) the extent of the growth as determined clinically; (3) the common directions of spread in similar cases as indicating regions likely to be infected, even though such infection is not clinically appreciable.

\section{Radio-Sensitivity of Malignant Growths}

Although in recent years considerable progress has been made in the histological classification of growths in relation to radio-sensitivity, our knowledge in this direction still leaves much to be desired. In general, the main histological varieties of growth have their special characteristics as regards sensitivity, but within the main groupings histology is of little or no help. Thus, a group of histologically similar breast carcinomas may vary greatly in their individual sensitivity. In order to simplify the discussion of this aspect of the problem I shall try to arrange the commoner growths in three groups: hiohly radio-sensitive, moderately radio-sensitive, and radioresistant.

1. Highly Radio-sensitive.-(a) Small, round-celled and lymphosarcoma; (b) seminoma; (c) some carcinomas of the breast ; $(d)$ some carcinomas of the ovary ; $(e)$ certain cerebral tumours-medulloblastoma.

2. Moderately Radio-sensitive.-(a) Round-celled sarcoma; (b) endosteal and chondrosarcoma ; $(c)$ some periosteal sarcomas; $(d)$ most carcinomas of the breast ; $(e)$ carcinoma of the prostate ; $(f)$ carcinoma of the bladder ; $(g)$ certain palatal, pharyngeal, and laryngeal growths-lympho-epithelioma; (h) hypernephroma ; (i) carcinoma of the cervix ; $(j)$ carcinoma of the corpus uteri ; $(k)$ carcinoma of the ovary ; $(l)$ carcinoma of the thyroid gland; $(m)$ carcinoma of the lip ; $(n)$ carcinoma of the skin.

3. Radio-resistant Growths.-(a) Carcinoma of the rectum; (b) carcinoma of the oesophagus ; (c) carcinoma of the tongue ; (d) many pharyngeal carcinomas ; $(e)$ some carcinomas of the breast ; $(f)$ some carcinomas of the ovary ; $(g)$ carcinoma of the bronchi; $(h)$ certain metastases in cervical glands, espe- cially those arising from carcincmas of the tongue ; (i) spindlecelled sarcoma and fibrosarcoma.

I would like to remind you, at this point, that radiosensitivity and curability are by no means synonymous terms. Indeed, the most radio-sensitive growths are frequently incurable. This is because the attack by radiation methods on a malignant growth is essentially a local one, and although multiple growths may be dealt with in the same patient with local success in every case, when the disease becomes generalized it is beyond the aid of radiotherapy. The known tendencies of the growth with regard to the formation of metastases must therefore be taken into account in forming a prognosis.

\section{Choice of Method of Irradiation}

We may now consider the choice of method of irradiation in these three groups of tumours.

\section{Highly Radio-sensitive Group}

When we say that a tumour is highly radio-sensitive we simply mean that it can be made to disappear by a dose of radiations which produces little or no damaging effects on normal tissues, local or remote. In this group the small round-celled sarcomas and lymphosarcomas, wherever they occur and whatever their extent, should invariably. b: treated by external irradiation, $x$ rays, or, perhaps, telecurietherapy. Of the two methods $x$-ray therapy is by far the more convenient and the more economical, and wide areas in the neighbourhood of the growth can be treated with ease and safety. The local results are entirely satisfactory, and unsatisfactory end-results in lymphosarcoma are due to generalization of the disease and not to failure to secure regression of local lesions. Moreover, in the absence of generalization, local recurrences are seldom encountered.

Interstitial methods should never be used in treating small round-celled sarcoma and lymphosarcoma. Their attack is much more local than that of $x$ rays, and from the structare of the growth the trauma of the insertion of the needles into its substance can only increase the risk of generalization. Seminomas of the testis should be treated in the same way as lymphosarcomas, and in these cases also the local results are entirely satisfactory. Even advanced recurrences and metastatic deposits from testicular growths can often be made to disappear for long periods-even years.

The highly sensitive carcinomas of the breast and of the ovary form only a small proportion of all cases of these conditions, and it is impossible to distinguish them in advance. All cases are therefore treated as the lesssensitive variety.

\section{Moderately Sensitive Group}

It is probably true that there is no case of malignant disease which would fail to yield to a sufficiently large dose of $x$ or gamma rays, and the limitations imposed on the success of our treatment are determined, therefore, by the vulnerability of the healthy tissues. By the term " moderately radio-sensitive" tumour we mean one which can only be destroyed by a dose of radiation which approaches fairly closely to the maximum tolerance dose of the healthy tissues. The treatment of these cases, by whatever method employed, therefore demands the exercise of great care and skill if severe damage to healthy tissues is to be avoided. For the moderately radiosensitive group of tumours, with the exception of carcinoma of the skin, I consider that interstitial radium is the best method of radiological treatment wherever the growth can be adequately dealt with by this means. By this method the most intense local effect can be produced with a minimum exposure of healthy tissues. Not only the growth, however, should be treated, but also the regional lymph glands associated with the affected region. 
If these can be adequately dealt with by interstitial radium also, this may be done. If, however, the glandular areas are not accessible to interstitial radium they should be irradiated by surface methods, either by $x$ rays or by surface radium. Short-distance surface radium may be used if the region to be treated is comparatively superficial, as in the neck. For deeper regions $x$ rays, or possibly telecurietherapy, should be employed.

Where growths in this group are too extensive to permit of adequate treatment by interstitial radium, treatment by $x$ rays in the first instance will often succeed in reducing the growth sufficiently to permit of the insertion of radium subsequently. This is an important point, inasmuch as it is far better to employ the combined method of treatment than a doubtfully complete interstitial radium treatment.

For the caśes in which, for anatomical and other reasons, interstitial radium cannot be applied $x$ rays should be used. In a proportion of these cases very good primary results are obtained, although, unfortunately, recurrence is only too common after a period of freedom lasting for months or years. Among the growths in which good results are frequently obtained by $x$ rays are certain pharyngeal and especially all palatal growths, carcinoma of the prostate, carcinoma of the cervix and corpus uteri, and carcinoma of the thyroid gland. In view of the tendency to recurrence sooner or later I would urge, however, that even when complete disappearance of the growth is effected by $x$ rays, either radium or surgery should be subsequently employed in the attempt to make the result more permanent.

The exceptions to these methods of treatment in the moderately radio-sensitive group are the carcinomas of the skin, which are best treated by $x$ rays. Surface radium may also be used, especially for the very small growths. The results obtained are entirely satisfactory when there is no involvement of bone or cartilage, even when the disease is extensive. When, however, bone or cartilage is involved the percentage of successes is very greatly reduced; this is also the case when previous incorrect radiation treatment has been applied. Skin growths that have been treated by repeated doses of soft or low-voltage $x$ rays for long periods may become quite radio-resistant.

\section{Radio-Resistant Growths}

The methods of treatment in this group are similar to those adopted in the moderately sensitive group, with the exception that the cases have to be more rigorously selected. Interstitial radium is the treatment of choice, but it is essential that the whole extent of the growth be thoroughly barraged and with full dosage. In cases in which this thorough irradiation cannot be effected $x$ rays may afford some degree of palliation, but they rarely achieve more. Perhaps the most difficult problem in this group is the treatment of the resistant cervical gland metastases, the proximity of the skin negativing the application of the very high doses which are necessary to secure regression of the growth. There is a tendency to return to block dissection whenever possible in these cases, with pre- and post-operative irradiation, either by $x$ rays or by surface radium.

Finally, a word about the combination of $x$ rays and surgery. In comparing the results of surgery and radiotherapy it is often found that in certain groups the threeor five-year results do not differ greatly. I do not think, however, that it is sufficiently realized that the factors which determine the success of radiotherapy and those which determine the success of surgery differ very greatly. It is likely, therefore, that even though the percentage of three-year or five-year successes may be the same, the individual cases that survive are probably quite different. Thus, in a particular group of cases treated by surgery,
Mrs. X might be the only survivor after five years, but if the same series of cases had been treated by radiotherapy the sole survivor might be quite a different person, Mrs. Y. In each case the percentage survival would be the same. The point I would urge is, can we by combining our radiotherapy with-surgery save Mrs. $X$ and Mrs. $Y$ too? It seems to me that in dealing with this deadly disease we should not be content with using only one means of treatment. Every proved means that is applicable should be applied. For example, even though we can make a breast growth disappear by means of radium, the breast still remains a potential source of danger to the patient and should be removed. It is difficult, I know, to advise a patient, who to all outward evidence is rid of her disease, to submit herself to a major operation, or, if she has already had a major operation, to an intensive irradiation. But there is, as yet, no method of treatment of malignant disease so satisfactory that we can afford to be lulled into a sense of false security by the contemplation of a satisfactory primary result.

\section{THE TREATMENT AND POST-OPERATIVE RESULTS OF PERFORATED PEPTIC ULCERS BY}

F. HECTOR SCOTSON, M.B., B.S., F.R.C.S.

HONORARY SURGEON, MANCHESTFR VICTORIA MEMORIAL JEWISH HOSPITAL

An investigation was made into the late results following operations performed for perforated peptic ulcers, and I wish to necord, in some detail, all relevant facts of a series of cases operated on by me during the years I acted as assistant resident surgical officer (May, 1925, to December, 1929) and resident surgical officer (June, 1927, to May, 1929) to the Manchester Royal Infirmary.

In my series there are 131 duodenal ulcers, forty-five gastric ulcers, and five gastro-jejunal ulcers. One of the gastro-jejunal ulcers followed a posterior gastro-jejunostomy, performed eleven years previously for a perforated duodenal ulcer, which was treated by suture and gastrojejunostomy; three followed anterior gastro-jejunostomy for duodenal ulcer; and one an anterior Polya gastrectomy for a gastric ulcer.

\section{SURVEY OF CASES}

SEX INCIDENCE AND History

There were 170 males and eleven females-a proportion of over 15 to 1 .

The usual large number of males as compared with the small number of females is evident in my survey.

TABLE I.-Sex Incidence according to Type of Ulcer

\begin{tabular}{rrrr|cr|c|c}
\hline \multicolumn{3}{c|}{ Sex } & & & Duodenal & Gastric & $\begin{array}{c}\text { Gastro- } \\
\text { jejunal }\end{array}$ \\
\hline Males & $\ldots$ & $\ldots$ & $\ldots$ & $\ldots$ & 125 & 40 & 5 \\
Females... & $\ldots$ & $\ldots$ & $\ldots$ & 6 & 5 & 0 \\
\hline Total & $\ldots$ & $\ldots$ & $\ldots$ & 131 & & 45 & 5 \\
\hline
\end{tabular}

This male sex preponderance, which is especially noticeable in the case of the duodenal ulcers, has been noted by Dineen, ${ }^{5}$ Hinton, ${ }^{8}$ Gilmour and Saint, ${ }^{6}$ and many others, and presents no new features.

\section{LENGTH OF HistoRY}

This varied greatly; in the case of the duodenal ulcers the length of history previous to the perforation of the ulcer ranged from one week to forty years-the average being :

$\begin{array}{lcccccc}\text { Males } & \ldots & \ldots & \ldots & \ldots & 32.38 \text { months } \\ \text { Females } & \ldots & \ldots & \ldots & \ldots & 85.17 & , .\end{array}$

